\title{
Biomarkers in Heart Failure
}

\section{Berezin $\mathrm{AE}^{*}$}

Senior Consultant of Therapeutic Unit, Internal Medicine Department, State Medical University of Zaporozhye, 26, Mayakovsky av, Zaporozhye, Ukraine

\begin{abstract}
Heart failure (HF) is considered a leading cause of death in patients with established cardiovascular (CV) and metabolic diseases. Although current treatment strategy has improved survival rate and clinical outcomes of HF, the HF prevalence exhibits growth especially in older patients' population and survivors after coronary atherothrombotic events. Current clinical guidelines regarding treatment and prevention of HF claim the role of biological markers as pretty easy and powerful tool for diagnosis, risk stratification, and prognostication of HF. However, there is not clear whether all these biological markers are able to equally predict CV death and HF-related outcomes in patients with acute and chronic HF as well as in various phenotypes of HF. The aim of the review is to discuss a role of in risk stratification and individual treatment in patients with different phenotypes of HF.
\end{abstract}

Keywords: Heart failure; Biomarkers; Prediction; Stratification; Biomarker guided-therapy

\section{Introduction}

Heart failure (HF) is considered a leading cause of premature cardiovascular $(\mathrm{CV})$ death in patients with established $\mathrm{CV}$ disease [1]. Prevalence of HF has been exhibiting a strong tendency to growth worldwide, despite the scientific progress in the field of the two past decades. HF is also characterized by an elevated rate of primary and secondary hospitalization and increased economic burden for patients and their families. Although there are pretty numbers of clinical guidelines, which clearly indicated diagnosis, prevention and evidencebased treatment of HF, a strategy regarding exclusion of HF diagnosis, as well as risk stratification of HF, nature evolution of disease is not well established and requires more development [2]. In this context, biological markers reflected several pathophysiological stages of HF have become a powerful and convenient noninvasive tool for diagnosis of HF, a stratification of HF patients at risk of progression, HF severity, and biomarker-guided therapy [3]. The aim of the review is to discuss a role of biomarker-based approaches for much more pretty accurate diagnosis, in-depth risk stratification and individual targeting in treatment amongst patients with HF.

\section{Conventionally Used Biomarkers of Heart Failure}

Currently updated clinical recommendations have been reported that the natriuretic Cs (NPs), including brain NP (BNP), mid-regional pro-atrial NP (MR-proANP), NT-pro-brain NP (NT-proBNP), midregional pro-brain NP (MR-proBNP), galectin-3, high-sensitivity cardiac troponins and soluble suppressor of tumorigenicity-2 (sST2) receptor are the most frequently used biomarkers in routine clinical practice to stratify patients at risk of HF development, a risk of admission/re-admission to the hospital due to HF-related reasons, and a risk of death (Table 1). Most data on cardiac biomarkers have been derived from chronic HF individuals. In contrast, risk prediction in patients admitted with ADHF remains a challenge.

\section{Natriuretic peptides}

First NPs were recommended by the European Society of Cardiology and American Heart Association for exclusion HF, and then they were discussed as a tool for risk stratification, and NPsguided therapy [2]. The majority of NPs' family members (Atrial NP $[\mathrm{ANP}]$ and brain [BNP] apart from C-type of NP (CNP) are mechanical stress-related markers. They are actively released by cardiomyocytes as a result in fluid overload, cardiac stretching, as well as due to exposure other causes, i.e., ischemia/necrosis, metabolic and toxic damage, membrane stability loss, and inflammation. In contrast, CNP is secreted from activated endothelial cells and renal cells in response to cytokines' activation and through endothelium-dependent agonists, i.e., acetylcholine. The biological effects of ANP and BNP are ensured by binding with appropriate NP receptor type A (NPRA). NPRA are expressed at the surfaces of the target cells and are cooperated with cGMP mediating water/electrolytes' homeostatic effects, i.e., wateruresis/natriuresis, increasing glomerular filtration rate, volume of circulating plasma, as well as suppressing systemic sympathetic activities, maintenance of cardiac output and regulation of blood pressure. Therefore, NPs may ensure anti-proliferative activity and anti-mutagenic effect, mediate vascular dilatation and prevent vascular wall hypertrophy. Additionally, NPs affect modest anti-aldosterone and endothelin-1 effects.

In patients with HF the plasma levels of BNP and NT-proBNP are typically $>100 \mathrm{pg} / \mathrm{mL}$ and $>250 \mathrm{pg} / \mathrm{mL}$, respectively, while there is high individual biological variability of both biomarkers irrespective presentation of HFrEF or HFpEF. Elevated levels of NPs are well correlated with clinical status and severity of HFrEF/HFpEF patients, a risk of acute HF/acutely decompensated HF (ADHF) regardless etiology of disease, risk of hospital admission/re-admission, as well as all-cause mortality, CV and HF death in individuals with established $\mathrm{HF}$ including at discharge period from the hospital after solving HF decompensation. Recently ended STOP-HF (The St Vincent's Screening to Prevent Heart Failure) trial [3] was reported that BNP-based screening was able to reduce the composite endpoint of asymptomatic cardiac dysfunction (regardless to systolic or diastolic) with or without newly diagnosed chronic HFrEF/HFpEF that confirms the immense role of screening NPs and early intervention may prevent HF. More recent evidence suggests that NPs along with the next generation of

*Corresponding author: Berezin AE, Senior Consultant of Therapeutic Unit Internal Medicine Department, State Medical University of Zaporozhye, Mayakovsky av, Zaporozhye, Ukraine, Tel: +380612894585; E-mail: aeberezin@gmail.com

Received June 02, 2017; Accepted July 27, 2017; Published July 31, 2017

Citation: Berezin AE (2017) Biomarkers in Heart Failure. J Blood Lymph 7: 172. doi: 10.4172/2165-7831.1000172

Copyright: (c) 2017 Berezin AE. This is an open-access article distributed unde the terms of the Creative Commons Attribution License, which permits unrestricted use, distribution, and reproduction in any medium, provided the original author and source are credited. 


\begin{tabular}{|c|c|c|c|}
\hline Suggestions for use & Patients & COR & LOE \\
\hline \multicolumn{4}{|c|}{ NPs (BNP, NT-proBNP or MR-proANP) } \\
\hline \multirow[t]{3}{*}{ Rule-in or support of initial working diagnosis } & Patients with suspected HF in non-acute setting condition with dyspnea & 1 & A \\
\hline & $\begin{array}{l}\text { Patients with suspected acute and chronic HF, when the etiology of dyspnea is } \\
\text { unclear }\end{array}$ & I & A \\
\hline & Patients with suspected $\mathrm{HF}$ in acute setting condition & lib & C \\
\hline Exclusion of important cardiac dysfunction & Outpatients with uncertain signs and symptoms of $\mathrm{HF}$ & 1 & A \\
\hline \multirow{3}{*}{ Prognosis of HF } & Outpatients/inpatients with established HF & I & A \\
\hline & Patients who admitted to the hospital with acutely decompensated HF & 1 & A \\
\hline & Postdischarged patients & lia & B \\
\hline $\begin{array}{l}\text { Prevent development of LV dysfunction or new-onset } \\
\text { HF }\end{array}$ & Patients at risk of HF development & lia & B \\
\hline Target therapy & Outpatients with established HF in euvolemic condition & lia & B \\
\hline \multicolumn{4}{|l|}{ Biomarkers of myocardial injury (cardiac troponins) } \\
\hline \multirow[t]{2}{*}{ Risk stratification } & Patients with established $\mathrm{HF}$ & I & A \\
\hline & Patients who admitted to the hospital with acutely decompensated HF & 1 & A \\
\hline \multicolumn{4}{|l|}{ Biomarkers of myocardial fibrosis (galectin-3) } \\
\hline \multirow[t]{3}{*}{ Risk stratification } & Outpatients with established chronic HF & lib & B \\
\hline & Inpatients with established acute and chronic HF & lib & A \\
\hline & Postdischarged patients & lia & B \\
\hline \multicolumn{4}{|l|}{ sST2 } \\
\hline \multirow[t]{3}{*}{ Prognosis of HF } & Outpatients/inpatients with established HF & I & A \\
\hline & Patients who admitted to the hospital with acutely decompensated HF & 1 & A \\
\hline & Postdischarged patients & lia & B \\
\hline
\end{tabular}

Abbreviations: HF: Heart Failure; NPs: Natriuretic Peptides; BNP: Brain Natriuretic Peptides; NT-proBNP: N-Terminal Fragment of Brain Natriuretic Peptides; sST2: Soluble Suppressor of Tumorigenicity-2; MR-proANP: Mid-regional Pro-atrial Natriuretic Peptides; COR: Classes of Recommendations; LOE: Level of Evidence. Table 1: Utility of biomarkers in HF management.

CV biomarkers could provide added predictive value to drug therapy of HF, which could potentially lower HF-related risk of outcomes $[4,5]$.

\section{Biomarkers of myocardial fibrosis}

Galectin-3: Galectin-3 is a soluble $\beta$-galactoside-binding protein, which is actively secreted by activated mononuclears and macrophages due to inflammatory stimulation. The main biological function of galectin-3 is to activate the fibroblasts for further collagen synthesis [6]. Recent pre-clinical and clinical studies revealed the pivotal role of galectin-3 in progressive accumulation of extracellular matrix leading to cardiac fibrosis, cardiac remodeling, and worsening cardiac performances associated with impaired systolic and diastolic function, dilation of cardiac cavities, and induce of cardiac arrhythmias [7]. Increased expression of galectin-3 was found in acute HF, ADHF, and chronic HFrEF/HFpEF regardless etiology of disease [8]. Moreover, galectin-3 in exaggerated concentrations was measured in a serum of the patients at risk of $\mathrm{HF}$ and $\mathrm{CV}$ disease [9]. In patients with acute HF and acutely decompensated HF galectin-3 was associated with NTproBNP levels and the estimated glomerular filtration rate (GFR) but not with age and serum cardiac troponins [10]. Nowadays galectin-3 is concerned the predictive biomarker for all-cause mortality, $\mathrm{CV}$ mortality and HF-related clinical outcomes in patients with established HF. Recent clinical trials have shown that galectin-3 was not superior to NT-proBNP, sST2 receptor, Growth Differentiation Factor (GDF)15 or high-sensitive C-reactive protein (hsCRP) in prediction of $\mathrm{CV}$ mortality and HF death. However the combination of NPs and galectin-3 was much more pretty accurate in predicting HF death compared to either of other biomarkers alone [11].

Soluble suppressor of tumorigenicity-2 receptor: Soluble suppressor of tumorigenicity-2 receptor (sST2) belongs to the interleukin (IL)-1 receptor family members, which was found in two comprising isoforms, i.e. membrane-bound (ST2L) and soluble (sST2) isoforms. ST2 interplays with its ligand IL-33 and through myocardial
mRNA expressions of Th1-related cytokines (tumor necrosis factoralpha) may directly enhance in cardiac hypertrophy, aggravating fibrosis, cavities dilation with impaired cardiac function. However, the IL-33/ST2 pathway is involved in the pathogenesis of HF across all pathophysiological stages of the disease and regardless its etiology. sST2 is posed as a cardiac mechanical strain biomarker having useful ability in independent prognostic stratification of HF patients. It has been found that serum levels of sST2 in acute HF/acutely decompensated HF were dramatically increased on admission and appeared to be decreased rapidly depending on clinical improvement. Therefore, sST2 in HF has well correlated with BNP and GDF15 levels [12]. Prognostic importance of sST2 was served for prediction of all-cause mortality, CV death and HF admission in HFrEF/HFpEF. However, sST2 levels at discharge were better predictor of HF re-admission than ones at admission. Although both biomarkers of myocardial fibrosis (sST2 receptor and galectin-3) are predictive of HF-related admission to the hospital and CV death [13], direct head-to-head comparison of sST2 and galectin-3 revealed superiority of sST2 over galectin-3 in HF risk stratification [14]. However, both biomarkers of fibrosis may provide better incremental prognostic value either NPs' levels in patients with HF.

\section{Biomarkers of myocardial injury}

There are some biomarkers of myocardial injury and necrosis (troponins $\mathrm{T}$ and $\mathrm{I}$, myoglobin, heart type of fatty acid binding protein, glutathione transferase $\mathrm{P} 1$ ), which are investigated in details as potential predictors of HF onset and HF-related outcomes [15]

Cardiac troponins: Since last two decades high-sensitivity cardiac troponins (troponins $\mathrm{T}$ and I) had been suggested to be prognosticators of higher risk of CV mortality and combined adverse CV outcomes in acutely decompensated or chronic HFrEF/HFpEF [16]. However, cardiac troponins releases from reversibly/irreversibly injured cardiomyocytes and have frequently found in elevated concentrations in patients with acute and chronic HF, there are speculative opinions 
and controversial evidence regarding their independent relation to acute HF outcomes [17]. Thus, more clinical investigations are required to clear the predictive role of these biomarkers in HF prediction and nature evolution.

Heart type of fatty acid binding protein: The main biological role of heart type of fatty acid binding protein (hFABP) is to facilitate the long-chain fatty acids re-uptake, attenuate calcium transport in cardiomyocytes and regulate inflammatory response in reply to some lipid signals [18]. hFABP is predominantly expressed in cardiomyocytes and is powerful biomarker of myocardial injury. Recent studies have shown that the hFABP has better predicted CV outcomes to other biomarkers of cardiac damage, i.e., myoglobin and high-sensitive cardiac troponins [19], whereas elevated intestinal FABP would identify patients with advanced HF who had severe fluid retention and intestinal congestion [20]. Overall, the hFABP may better provide prognostic information on survival and more precise reflect a risk of major $\mathrm{CV}$ events during hospitalization period and short-time after discharge than natriuretic peptides, cardiac troponins and galectin-3. However, the role of several types of FABP in HF is not fully clear. Large clinical studies are required to more accurately explain the predictive value of these biomarkers especially in head-to-head comparison with other molecules, i.e., myoglobin, and glutathione transferase P1.

\section{Biomarker(s)-based Strategies of Pharmacological and Non-pharmacological Therapies}

Biomarker(s)-guided therapy with serial biomarker values is considered a pretty reliable and as it is suggesting effective method for timely therapeutic adjustment in HF management. Although there are some speculations regarding strong evidence of biomarkerguided HF therapy, the proof-of-concept appears to be promising for individualizing medical care including rehabilitation methods in HF.

\section{Biomarker-based HF-guided therapy}

As it had been suggested the biomarker(s)-guided HF therapy could improve a routine clinical management through adjusted doses/routes of drug(s) and increase a competence regarding decision-making for an admission to the hospital before urgent state onset. Indeed, NP guided HF therapy improves titration of medications. However, taking into consideration the results of recently completed multi center randomized clinical trials there has not obviously become whether biomarker(s)-guided therapy would associate with better HF clinical outcomes during average 6-12 month follow-up [2]. Meanwhile, serial measurements biomarkers could be useful for determining severity of HF for interference of ambulatory and in-hospital medical care. Additionally, NT-proBNP, but not BNP, is better suited during HF therapy based on the new angiotensin-receptor-neprilysin-inhibitor (ARNI). Indeed, new era in use of NPs in monitoring of HF evolution has been opened after implementation in the routine clinical practice [21]. Recent clinical trials have been shown that nephrilisin inhibition auxiliary to chronic renin-angiotensin system blockage with LCZ696 (Sacubitril/Valsartan) may increase the bioavailability of NPs and promotes additional benefits the cardio-renal system and hence protected against all-cause mortality, CV mortality and HF death [22]. Because of biologically active BNP is degraded by neprilysin, in HF patients treated with ARNI circulating level of BNP sufficiently increases, whereas NT-proBNP concentration declines dramatically. On this occasion, the principles of NPs-based HF guided therapy are challenging. Apparently, monitoring of BNP levels is not suitable for risk stratification and HF adjusted medical care, when ARNIs are used, however, NT-proBNP remains to be a main key for initiated risk assessment and appraised HF stratification regardless drugs' prescription [23].

There are expectations regarding that the galectin-3- and procalcitonin-based HF therapies would be better than NP-guided treatment strategy in HFrEF/HFpEF. However, there is no strong evidence for clinically-proven data about this conception because there are findings for suboptimal sensitivity and/or specificity of HF management [24].

\section{Biomarker-based cardiac rehabilitation programs}

There is a large body of evidence regarding that NT-proBNP, galectin-3, sST2, MR-proADM, and mid-regional pro-adrenomedullin (MR-proANP) could have much more prognostic importance for cardiac rehabilitation programs in HF individuals [13,25]. It has suggested that an overall improvement in the neuro-hormonal profile due to cardiac rehabilitation may correspond to increase of survival probability, rather in patients with HF with reduced ejection fraction (HFrEF) than in individuals with HF with preserved ejection fraction (HFpEF). Finally, majority of experts believe that a combination of biomarkers may ultimately prove to be more informative in their predictive ability than single biomarker, while this issue is pretty discussable.

\section{Limitations in Use of Conventional Biomarkers in HF}

Confusingly, the role of NPs in modification of treatment care considerably relates to aging, $\mathrm{CV}$ disease and metabolic comorbidities, kidney clearance, metabolism (neprilysin for BNP, glycosylation, methylation, oxidation for other NPs), toxic effect (cardiotoxicity). Therefore, higher individual biological variability of these biomarkers, which negatively effects on interpretation of measure results. Additionally, there is a huge list of the diseases associated with increased level of NPs beyond HF development (Table 2).

Although galectin-3 is an independent predictor of all-cause mortality, CV death and occurrence of HF, there is an inverse relationship between serum galectin-3 and estimated glomerular filtration rate. Accordingly, lowered kidney clearance should be taken into consideration, when data of galectin-3 measurement are interpreted. Therefore, older patients contributed to higher galectin-3 concentrations than younger individuals. Amongst other biomarkers (NPs, GDF-15, high-sensitivity troponin T, sST2, aldosterone, phosphate, parathyroid hormone, plasma renin concentration, and creatinine) galectin-3 had the lowest indices of individual biological variability, whereas NPs and GDF-15 has the highest ones. Additionally, in contrast to NPs serum galectin-3 levels did not appear to be significantly related to circulating level of cardiac troponins, left ventricular (LV) ejection fraction and LV mass index. Therefore, there was a positive correlation galectin-3 levels with NT-proBNP in HF individuals. Thus, galectin 3 and NPs might be allocated as the best tool for both short- and long term death prediction in HF regardless kidney function and age. Unfortunately, no one biomarker predicted the shortterm composite HF endpoints in acute HF and actually decompensated chronic HF. Additionally, there are controversial findings regarding that there was no association of galectin-3 concentration with adverse outcomes in chronic HF.

Optimistically results of recent clinical trial about higher predictive value of sST2 receptor in HF have associated with some evidence regarding that sST2 was related to increased age, female sex, and some comorbidities including diabetes, atrial fibrillation, inflammatory diseases, kidney insufficiency and myocardial infarction. 


\begin{tabular}{|c|c|c|c|}
\hline \multirow{2}{*}{ Diseases } & \multirow{2}{*}{$\begin{array}{l}\text { Direction to } \\
\text { changes }\end{array}$} & \multicolumn{2}{|c|}{ Causes for NP evolution } \\
\hline & & Primary & Other \\
\hline Acute and chronic HF & $\uparrow \uparrow \uparrow$ & $\begin{array}{l}\text { Over-production due to myocardial wall stretching/fluid } \\
\text { overload }\end{array}$ & Lowered kidney clearance, cardiac injury \\
\hline MI/ACS & $\uparrow \uparrow$ & Cardiac injury & Fluid overload, biochemical stress, ischemia/hypoxia \\
\hline Atrial fibrillation/atrial flutter & $\uparrow \uparrow$ & Leakage through cardiac myocyte membrane & Cardiac injury \\
\hline Myocardities/cardiomyopathy & $\uparrow-\uparrow \uparrow \uparrow$ & Cardiac injury & $\begin{array}{l}\text { Leakage through cardiac myocyte membrane due to } \\
\text { inflammation, fluid overload, biochemical stress }\end{array}$ \\
\hline Cardiac hypertrophy & $\uparrow$ & Leakage through cardiac myocyte membrane & Biochemical stress \\
\hline Cardioversion & $\uparrow$ & Cardiac injury & Metabolic myocardial damage \\
\hline Cancer chemotherapy & $\uparrow$ & Toxic-metabolic myocardial insults & Biochemical stress \\
\hline Valvular and Pericardial disease & $\uparrow-\uparrow \uparrow$ & Leakage through cardiac myocyte membrane & Biochemical stress, fluid overload, cardiac injury \\
\hline Pulmonary hypertension & $\uparrow-\uparrow \uparrow$ & Leakage through cardiac myocyte membrane & Fluid overload, biochemical stress, ischemia/hypoxia \\
\hline Cardiac surgery & $\uparrow$ & Leakage through cardiac myocyte membrane & Biochemical stress, fluid overload, cardiac injury \\
\hline Aging & $\uparrow$ & Lowered kidney clearance & Biochemical stress \\
\hline DM & $\uparrow-\uparrow \uparrow$ & Lowered kidney clearance & Cardiac injury, fluid overload, biochemical stress \\
\hline COPD & $\uparrow \uparrow$ & Myocardial wall stretching & Fluid overload, cardiac injury \\
\hline Obesity & $\downarrow$ & $\begin{array}{l}\text { Increased degradation by enzymes (glycosylation for } \\
\text { NT-poBNP, nephrylisin for BNP) }\end{array}$ & Increased kidney clearance \\
\hline Anemia & $\uparrow$ & Leakage through cardiac myocyte membrane & $\begin{array}{l}\text { Metabolic myocardial damage, biochemical stress, } \\
\text { cardiac injury, ischemia/hypoxia }\end{array}$ \\
\hline Renal failure & $\uparrow$ & Lowered kidney clearance & Biochemical stress, metabolic myocardial damage \\
\hline $\begin{array}{l}\text { Critical illness, bacterial sepsis, } \\
\text { severe burns }\end{array}$ & $\uparrow-\uparrow \uparrow$ & Lowered kidney clearance & $\begin{array}{l}\text { Metabolic myocardial damage, biochemical stress, } \\
\text { cardiac injury, ischemia/hypoxia }\end{array}$ \\
\hline
\end{tabular}

Abbreviations: NP: Natriuretic Peptide; HF: Heart Failure; ACS: Acute Coronary Syndrome; MI: Myocardial Infarction; COPD: Chronic Obstructive Pulmonary disease; DM: Diabetes Mellitus.

$\uparrow:$ Mild Increase; $\uparrow \uparrow:$ Moderate Increase; $\uparrow \uparrow \uparrow:$ Severe Increase; $\downarrow$ : Decrease.

Table 2: The potential causes of changes in circulating NPs' levels.

Additionally, sST2 was not associated with LV structure or LV systolic or diastolic function. Thus, these findings confirmed that the sST2 is rather a systemic inflammatory marker of extra cardiac origin of $\mathrm{HF}$ deteriorations than a single prognosticator of HF evolution; while meta-analysis provided by Anguita [15] pointed that sST2 level may predict all-cause and CV death in HF patients at admission and at discharge from the hospital. Overall, there is a large body of evidence regarding that improved discriminative value of multiple biomarkers in HF patients requires much more accurate confirmation. In this context, novel biomarkers are needed to improve in prediction models and assist in the titration of medical therapy.

\section{Novel Biomarkers for HF Management}

The discovery of new biomarkers remains to be promised, but rarely novel molecules prove to be significantly better in diagnostic and predictive manner than established biomarkers. However, additionally to various types of NPs, galectin-3, sST2, and highly sensitive cardiac troponins, multiple other biomarkers, including those of inflammation, oxidative stress, vascular dysfunction and reparation state, biochemical myocardial stress and matrix remodeling, have been implicated in HF management (Table 3). Additionally, there is no strong and clear evidence regarding that the new biomarkers are able to predict clinically significant end-points (i.e., all-cause and CV mortality, HF admission/ re-admission, and HF death) in both HF phenotypes - HFpEF and HFrEF. Recent clinical trials have been revealed that majority of new biomarkers indicated rather HF phenotype-related clinical outcomes than independently predicted any end points regardless presentation of HFpEF/HFrEF. Probably, biomarker-based approach could be useful to characterize pathophysiological differences between HFrEF and HFpEF patients.

\section{Inflammatory biomarkers}

C-reactive protein: The high-sensitive C-reactive protein (hsCRP) is well-established independent predictor for adverse $\mathrm{CV}$ outcome including CV death, all-cause mortality, sudden cardiac death, and HF-related death in general population, patients at higher $\mathrm{CV}$ risk and amongst individuals with known CV disease. Recent clinical studies have shown that the levels of hs-CRP were considerably higher in HFpEF than in HFrEF and independently associated with HFrEF development. Moreover, in HFrEF patients serum hs-CRP levels have positively correlated with circulating NT-proBNP and inversely with left ventricular ejection fraction (LVEF). In contrast, low to moderate hs-CRP levels did not exhibit an association with HFpFF, while they had to be going to support CV risk.

Calprotectin: Calprotectin (myeloid-related protein 8/14) is an inflammatory marker, which has been found elevated in patients suffering from cardiac conditions, e.g., myocardial infarction, unstable angina and HF. Calprotectin is predominantly expressed in activated human neutrophils, monocytes, adipocytes, and innate immunity cells including macrophages, but not in normal tissue macrophages. Calprotectin binds with Toll-like receptor 4 and acts as innate amplifier of infection, autoimmunity, and cancer. Although calprotectin was found as a nonspecific marker for atherosclerosis, kidney damage, vascular complications in metabolic disease including vascular calcification and endothelial dysfunction, it role in HF is not understood. It was established that patients with chronic HF regardless of LVEF had significantly higher levels of calprotectin than patients without HF. However, predictive value of calprotectin requires to be conformed in future.

Procalcitonin: Procalcitonin is known a precursor of the calcitonin, which is produced and actively secreted by the parafollicular $\mathrm{C}$ cells of the thyroid gland and involved in regulation of calcium 


\begin{tabular}{|c|c|c|}
\hline $\begin{array}{l}\text { Related pathophysiological } \\
\text { processes in HF }\end{array}$ & Biomarkers & Relevance to clinical outcomes in HF \\
\hline \multirow[t]{5}{*}{ Myocardial biochemical stress } & MR-proANP & All-cause, CV and HF-related mortality, risk of hospital re-admission at discharge, risk of HF deterioration \\
\hline & Copeptin & All-cause and HF-related death, CV mortality, hospital admission rate \\
\hline & CT-proET-1 & NYHA stage \\
\hline & ADM/MR-proADM & All-cause mortality, CV mortality and HF-related death in acute HF, ADHF, HFrEF \\
\hline & GDF-15 & Prediction of HFrEF, CV mortality, HF deterioration \\
\hline \multirow[t]{4}{*}{ Myocardial fibrosis } & $\mathrm{PICP}$ & AF, CV mortality, MI, HF-related death \\
\hline & CITP & AF, CV mortality, MI, HF-related death \\
\hline & PIIINP & All-cause mortality, CV mortality, MI, HF-related death \\
\hline & MMPs & All-cause, $\mathrm{CV}$ and HF-related mortality in acute HF, ADHF, risk of HF admission in HF \\
\hline \multirow[t]{2}{*}{ Myocardial necrosis } & hFABP & CV and HF-related mortality \\
\hline & GSTP1 & MI mortality, CV events and HF admission \\
\hline \multirow[t]{3}{*}{ Vascular remodeling } & OPN & CV mortality, MI, HF onset \\
\hline & OPG & CV mortality, MI, HF onset \\
\hline & miRNAs & All-cause and CV mortality, MI, HF onset, HF progression \\
\hline \multirow[t]{2}{*}{ Inflammation } & hs-CRP & NYHA stage of HF, risk of death in ADHF \\
\hline & Procalcitonin & ADHF, acute HF, CV death, readmission rate \\
\hline \multirow[t]{5}{*}{ Oxidative stress } & Uric acid & All-cause and CV mortality in HFrEF \\
\hline & Myeloperoxidase & All-cause and CV mortality in ADHF, acute HF, HF-related outcomes in chronic HF \\
\hline & Ceruloplasmin & Risk of HF deterioration, NYHA-stage \\
\hline & 8-OHdG & Risk of HF deterioration, NYHA-stage \\
\hline & Trx1 & Risk of HF deterioration, NYHA-stage \\
\hline \multirow[t]{2}{*}{ Renal dysfunction } & Cystatin C & All-cause and CV mortality, HF-related death, HF readmission in acute HF, ADHF, HFrEF \\
\hline & NGAL & HF-related death in acute HF and ADHF \\
\hline \multirow[t]{2}{*}{ Endothelial dysfunction } & EPCs & \multirow[t]{2}{*}{ All-cause mortality, CV mortality, HF-related death, admission/readmission rate } \\
\hline & EMPs & \\
\hline
\end{tabular}

Abbreviations: ADHF; Acutely Decompensated Heart Failure; MR-proANP: Mid-Regional pro Atrial Natriuretic Peptide; ADM: Adrenomedullin; MR-proADM; Mid-Regional pro-Adrenomedullin; PICP: Carboxy Terminal Propeptide; CT-proET-1: C-Terminal-pro-Endothelin-1; CITP: Carboxy-Terminal Telopeptide; PIIINP: Amino-Terminal Peptide of Procollagen Type III; AF: Atrial Fibrillation; H:, Heart Failure; hFABP: Fatty Acid Binding Protein; GDF: Growth Differentiation Factor; EPCs: Endothelial Progenitor Cells; EMPs: Endothelial Cell-Derived Micro Particles; MI: Myocardial Infarction; MMP: Matrix Metalloproteinase; NGAL; Neutrophil Gelatinase-Associated Lipocalin; 8-OHdG, 8-Hydroxy-2'-Deoxyguanosine; Trx1, Thioredoxin 1; GSTP1; Glutathione Transferase P1.

Table 3: Novel biomarkers for HF management.

homeostasis. Recent clinical studies have shown that procalcitonin as an inflammatory biomarker had a pretty accurate diagnostic ability to sepsis, shock, bacterial complications of some diseases. Additionally, this biomarker may help to manage the patients with HF when antibiotic use is needed or the critical state has been verified. However, there is not strong evidence regarding procalcitotin use in biomarkerguided therapy to adjust dosage of drugs for HF individuals.

\section{Biomarkers of biochemical myocardial stress}

Copeptin: Copeptin is C-terminal peptide derived from the precursor molecule of arginine vasopressin, which plays a pivotal role in fluid retention and electrolyte homeostasis. In the general population elevated level of copeptin strongly associated with increased CV mortality. Additionally, based on results of serial measurements of copeptin level it has been suggested that the increased copeptin concentration or trend to elevation of one are an independent risk factor for long-term HF-related clinical outcomes and sudden death in patients with established CV disease. Being able to better predict all-cause mortality rate and HF-related risks including death and admission to the hospital copeptin might be considered as much more accurate biomarker than NPs for optimize medical care in HF patients. Unfortunately, there are large body of evidence regarding that the level of copeptin might relate closely to some metabolic abnormalities including hyperglycemia that sufficiently limits the predictive power of the biomarker in serial measurements especially in patients with diabetes mellitus and abdominal obesity. However, the improvement of diagnostic reliability of copeptin may achieve by means use of combined biomarker strategy, in particular it might be based on copeptin and NPs. Finally, circulating level of copeptin has now recognized a promising biomarker with better discriminative value for both all-cause mortality and HF-related outcomes general population and individuals with established CV disease.

Growth differentiation factor-15: Growth differentiation factor (GDF)-15 belongs to the superfamily of transforming growth factor- $\beta$. GDF-15 is widely expressed on the surfaces of various cells. In HF GDF-15 is secreted by injured cardiomyocytes in response to ischemia, reperfusion, inflammatory cytokine stimulation and exposure to biomechanical stress. Elevated level of circulating GDF-15 was found in $\mathrm{HF}$ individuals irrespectively etiology of cardiac dysfunction. There is strong evidence regarding being tight interrelationship between circulating level of GDF-15 and HF signs and symptoms, reduced LVEF. Although serial biomarker evaluation has not showed superiority of incremental predictive ability in GDF-15 versus NPs in acute HF, in chronic HFrHF/HFpEF biomarker strategy based on GDF-15, galectin-3 and NPs might exhibit several advantages before conventional approach in ability to predict all-cause mortality, CV mortality and HF-related outcomes in outpatients with HF.

\section{Endothelial cell-derived micro particles and endothelial progenitor cells}

Impaired endothelial function plays a pivotal role in the $\mathrm{HF}$ development and HF-related complications and also associates with appearance in peripheral blood specific circulating biomarkers, i.e. endothelial microparticles (EMPs) and endothelial progenitor cells (EPCs). EPCs are involved in the repair of vascular wall and 
myocardium and, therefore, their ability to restore integrity and functionality of vasculature strongly relates to EMPs. EMPs are not only cargo for cell-to-cell transfer of variety molecules (i.e. peptides, DNAs, RNAs, active molecules, growth factors and hormones), but they are independent regulators of immunity, inflammation, reparation, proliferative response and malignancy. Number, origin (received from activated cells or apoptotic cells) and immune phenotypes of EMPs can be key factors in ensuring function of endogenous repair system. Thus, EPCs and EMPs are epigenetic co-regulators of vascular function playing a pivotal role in maintenance of endothelium integrity across all stages of HF. Recent clinical studies have shown that an ability of mature endothelial cells and their precursors to release of secretom progressively worse depended on HF stage and severity. Moreover, increased number of apoptotic EMPs and decreased number of EPCs in circulation has found as powerful predictor of CV death, HF-related admission to the hospital and prognosticator of positive reply to medical therapy in short-term period. There is novel HF risk prediction score created by means of biomarkers including NPs, galectin-3, high sensitive CRP and estimated ratio between both numbers of apoptotic EMPs and EPCs. However, there is not clear whether new predictive model would be effective in discriminative concern of HF treatment. More clinical trials are required to improve our understanding in the field of individualized therapy of HF under biomarker control.

\section{Biomarkers of collagen metabolism}

Recent studies have shown that impaired collagen metabolism may alter the myocardial collagen network and thereby it exerts cardiovascular remodeling, promotes the fibrotic substrate and mediates HF complications, i.e. atrial fibrillation/flatter, sudden death, and declining LV pump function. Altered collagen type I synthesis and advance in degradation of one associated with appearance of circulating biomarkers, i.e., carboxy-terminal propeptide (PICP), amino-terminal peptide of procollagen type III (PIIINP) and carboxyterminal telopeptide (CITP). Interestingly, there is evidence regarding direct causative role of BNP in alterations in collagen type I metabolism in HFrEF. In the OPTIMAL (The Optimizing Congestive Heart Failure Outpatient Clinic trial) was found that disturbances of collagen type I metabolism have exhibited an independent prognostication for longterm all-cause mortality and CV mortality in HFrEF individuals. In the Cardiovascular Health Study $(n=880)$ in which was included 146 patients with HFrEF (LVEF <55\%) 175 patients with HFpEF (LVEF $\geq 55 \%$ ), 280 individuals with traditional CV risk factors without chronic HF, and 279 healthy and elderly volunteers with CV disease at risk, biomarkers of collagen turn-over (PIIINP and CITP) were significantly associated with $\mathrm{CV}$ outcomes, i.e. death, myocardial infarction, and advanced HF. Therefore, circulating CITP is probably independent predictor of survival in patients with HFrEF. Moreover, CITP level added to NT-proBNP level exhibited an additive predictive value compared with NT-proBNP level alone. The estimated negative predictive value for both biomarkers for long-term $\mathrm{CV}$ outcomes was 94\%. Thus, biomarkers of collagen turn-over might be a powerful component for novel multi-marker strategy for risk stratification of HF.

\section{Biomarkers of cardiovascular remodeling}

Matrix metalloproteinase: Adverse cardiac remodeling strong relates to an accumulation of non-fibrillar extracellular matrix (ECM) and matricellular proteins, which contribute to disease progression. In fact, matrix metalloproteinases (MMPs), which are modulated by biomechanical and oxidative myocardial stress, neurohormonal and inflammation, essentially determine extracellular reposition of collagen and mediates pro-fibrotic process. According to current understanding in HF the MMPs correspond to an immune activation, inflammation, cardiac injury/vascular dysfunction to maintain tissue structure and metabolism. MMPs play a pivotal role in cardiac and vascular remodeling through enhancing cell-to-cell interactions acting as regulator of cell growth, proliferation, differentiation, survival, and migration. However, the pathogenetic role of MMPs in HF appears to be uncertain and could relate to etiology of cardiac dysfunction. Resent pre-clinical and clinical studies have shown that impairment of cardiac function may relate to collagen accumulation due to imbalance between expression of MMPs, predominantly MMP-1, MMP-3, MMP6, MMP-9, and suppression of their tissue inhibitors. However, the predictive potency of these biomarkers did not confirm and requires more investigations in future.

Bone-related proteins: Bone-related proteins (BRPs) belong to the family of matricellular proteins, which incorporate into extracellular matrix and play in the bone developing, vascular remodeling, and tissue regeneration. Amongst family of BRPs osteopontin (OPN), osteoprotegerin (OPG), osteonectin (OSN), osteocalcin (OCN), sclerostin, and some components of RANKL/RANK system are the most known. Overall, all these molecules are mediators for paracrine signaling in cell metabolism and extracellular matrix regulation that relate inflammation with epigenetic regulation of cell function. The role of BRPs in CV disease including HF is controversial. As multiple functional growth factors some members of the BRPs' family are able to cause cell differentiation and growth, bone and ectopic calcification, vascular remodeling, atherosclerotic plaque shaping, angiogenesis and neovascularisation acting predominantly in result in a hypoxic/ ischemia, metabolic, oxidative and inflammatory stimuli. On the other hand, BRPs may prevent cardiac dysfunction, hypertrophy and fibrosis through blocking cellular signaling systems (i.e., PI3K and Akt phosphorylation), reduced expression of extracellular matrix and hypertrophy genes. Although OPN, OPG, and RANKL/RANK components are well-known biomarkers of vascular calcification, systemic inflammation, atherosclerosis, kidney dysfunction, and cardiac remodeling, their predictive role in HF is still uncertain. There is evidence regarding that the sRANKL/OPG complex may relate to HFrEF development, whereas circulating levels of OPN and OPG corresponded to HFpEF. Moreover, an expression of OPN, OPG and OSN genes in myocardium or vasculature sufficiently distinguished in HFrEF and HFpEF and thereby it has been suggested that BRPs could be markers to suggest the development of different HF phenotypes. Indeed, in the PEACE trial levels of OPN were independently associated with the composite CV outcomes and incident HFpEF hospitalization. Finally, OSN, OPN and OPG have exhibited the predictive value to mortality rate in HFpEF regardless of its etiology, while there was no axillary discriminative effect in entire predictive score when these biomarkers were added to the NPs, hs-CRP, galectin-3 and sST2.

Other biomarkers of vasculogenesis: Although vascular endothelial growth factors (VEGF) act through appropriate receptors A and B, neurophilin may bind some VEGF molecules and contributes in vascular reparation. Thus, both factors are important components of endogenous repair system. Noted, that linear regression followed by network analyses revealed prominent inflammation and angiogenesisassociated interactions through VEGF-related mechanisms in HFpEF and mainly myocardial stretch-associated interactions in HFrEF. The neuropilin has demonstrated a predictive value for all-cause mortality and HF-related re-admission at 18 months in HFpEF, but not in HFrEF. Overall, the role of VEGF-related biomarkers in prediction of $\mathrm{HF}$ is not clear and needs to be explained in details in future. 


\section{Biomarkers of oxidative stress}

Serum uric acid: Observational and clinical studies have shown that the elevated serum uric acid (SUA) is common feature for patients with CV disease including HF, hypertension, atherosclerosis, obesity, diabetes mellitus and chronic renal disease. Evidence regarding the role of SUA in pathogenesis of CV disease is controversial. On the one hand, SUA attenuates oxidative stress through overproduction of reactive oxygen species and consequently it often worse vascular/endothelial function indirectly via inflammatory damage, inducing vascular calcification and directly via cell membranes deterioration effect. On the other hand, low-grading inflammation that is frequently found in HF may cause xanthine oxidase over-activity and leads to increased tissue SUA accumulation, which acts as scavenger of free radicals and protects against an damage effect of oxidative stress. Additionally, an increase of SUA may be an attribute of lowered kidney clearance as a result in a progress of HF. Therefore, there is evidence regarding the regulatory role of SUA in EPC mobbing and differentiations that allow discussing SUA as mediator of reparation of tissue in HF. Numerous clinical studies have emphasized the predictive role of baseline SUA for early post-discharge HF outcomes. Although levels of SUA did not significantly changed for admission period in HF patients, SUA at admission could be considered as powerful prognosticator of ADHF. On the other hand, an elevated SUA level on admission in patients with acute HF or ADHF associated not only with HF severity, but with the presence of chronic renal disease and the use of loop diuretics, which are able to cause negative clinical outcomes and independent predictor of 1-year mortality through elevation of SUA. Interestingly, the activity of xanthine oxidoreductase that is a key rate-limiting enzyme of purine degradation may be more accurate predictor of HFrEF severity and HF clinical outcomes than SUA. Consequently, SUA remains a well-known risk factor of HF related clinical outcomes in acute HF and ADHF, while poor prognosis in patients with both phenotypes of chronic HF (HFrEF and HFpEF) is not elucidated.

Other biomarkers: Serum levels of myeloperoxidase, ceruloplasmin and 8-hydroxy-2'-deoxyguanosine closely correlated with stage of chronic HF regardless LVEF and predict a development of HFrEF, while the role of these biomarkers of oxidative stress remains under scientific discussion and requires more investigations.

\section{Biomarkers of renal dysfunction}

Cystatin C: Cystatin $\mathrm{C}$ is an endogenous inhibitor of cysteine proteases and this biomarker is widely discussed an alternative predictor of $\mathrm{CV}$ events in acute and chronic HF patients with any types of cardiorenal syndrome. The patients with HFrEF demonstrated elevated serum cystatin $\mathrm{C}$, especially in cases with serious risk of $\mathrm{CV}$ complications. Additionally, in hypertensive patients with HFpEF increased cystatin C level was found. Therefore, it associated with LV diastolic dysfunction and alterations in collagen metabolism regardless of estimated GFR. Although cystatin $\mathrm{C}$ has now validated a powerful predictor of $\mathrm{CV}$ outcomes and kidney injury, its sensitivity in patients with chronic HF is sufficiently inferior to that of hs-CRP and NPs. In contrast, in acute HF and ADHF Cystatin C provided an incremental value for prognosis more than NT-proBNP and SUA.

Other biomarkers of kidney injury in HF: There is a large body of perspective biomarkers of kidney injury that could be useful for stratification of $\mathrm{HF}$ at risk, i.e. stromal cell-derived factor-1, exosomes, MPs, neutrophil gelatinase-associated lipocalin, kidney injury molecule-1, interleukin-18 and miRNAs. Although they are able to emerge at the early stages of renal dysfunction prior to any elevations in serum creatinine, the prognostication of clinical outcomes due to acute $\mathrm{HF}, \mathrm{ADHF}$ and chronic HF require more investigation.

\section{Genetic biomarkers}

By now, genetic testing has incorporated as a part of patient evaluation for suspected inherited cardiomyopathies. It turns out the epigenetic modifications through DNA methylation, ATP-dependent chromatin remodeling, histone modifications with an involvement of microRNA-related mechanisms might be sufficient pathophysiological factors contributing to adverse cardiac remodeling and altered cardiac function. In this context, the novel risk scores reflecting variabilities in genetic and epigenetic features in HF development appear to be promised. Indeed, some early studies have reported interested results with respect to genetic precursors of HFpEF and HFrEF. As biomarkers particularly used to scrutiny single nucleotide polymorphisms (SNPs) of genes encoding enzymes related to oxidative stress, genotype of guanine nucleotide-binding proteins (G-proteins) beta-3 subunit (GNB3), transcription factor Islet-1 gene, troponin T, CYP2D6 polymorphism, cardiac myosin binding protein- $\mathrm{C}$ mutations, reninangiotensin-aldosterone system polymorphism etc. Indeed, it is well known that angiotensin-converting enzyme (ACE) I/D gene D allele was associated with higher overall mortality as compared with the I allele in HF patients and that the effect could be modified by ACE inhibitors. Additionally, ACE DD and angiotensin-1-receptor 1166 CC genotypes may synergistically increase the predisposition to HFpEF.

Unfortunately, in ARIC (Atherosclerosis Risk in Communities) study was reported that none of the metabolite SNPs including pyroglutamine, dihydroxy docosatrienoic acid were individually associated with incident HF, whereas a genetic risk score created by summing the most sufficient risk alleles from each metabolite determined $11 \%$ greater risk of HF per allele. Luchner et al. (23) have reported that amongst 707 common SNPs associated with 125 diseases including HF it would not be easily obtained explainable results by common genetic variants related to HF development. Consequently, a close gene-gene interaction may determine an individual risk to development of HF through different pathways including epigenetic modifications. All these findings lead to assume that genes score might be a powerful tool for prediction of HF development.

More successful genome-wide linkage studies toward genes-related contribution in HF have been devoted incorporating SNPs of several genes (i.e., the bradykinin type 1 receptor gene, angiotensin-II type I receptor gene, the $\beta 1$-adrenoceptor gene and CYP2D6 polymorphysm) in predictive score to benefit and suffer harm from HF therapy. Although these parmacogenetic studies have focused on promised topics, the obtained results have not been absolutely consistent. Aspromonte et al. (24) have found no sufficient association between pharmacogenetic scores and fatal outcomes in HF patients. In contrast, Nakanishi et al. (25) have guessed that the gene expression profiling might be useful rather for risk prediction in HF than for choosing HF treatment regime. Thus, the clinical implementation of the HF therapy based on genes scoring remains uncertain and requires more evaluation in the future.

\section{Multiple Biomarker Predictive Scores}

Multiple biomarkers' use strategies based on the combination of NPs with other biomarkers have been discussed as priority in creating of much more accurate predictive scores in HF. Although there are several predictive scores based on biomarker measurement and approved for chronic HF, predictive scores for and acutely decompensated HF 
have not been validated. Current multiple biomarker score toward prognostication, risk stratification and diagnosis of HF is based on NPs in combination with biomarkers of myocardial injury and fibrosis (galectin-3 and sST2 receptor). It is validated by American Heart Association/American College of Cardiology at 2017 and the score is suitable for patients at risk of HF, individuals with established chronic HF (for both HFrEF and HFpEF), patients with suspected acute HF and documented acute/acutely decompensated HF, as well as patients with $\mathrm{HF}$ at discharge from the hospital. However, there is need to compare novel scores with recently created and the scores used in HFrEF and HFpEF to optimize the treatment approach in HF management.

Recently developed biomarkers, i.e., mid-regional pro-A-type natriuretic peptide (Mid Pro-ANP), mid-regional-proadrenomedullin (MR-proADM), pro-endothelin, and copeptin, when were added to the predictive model based on well-known prognostic biomarkers (NPs, troponin, hs-CRP, pro-calcitonin), have been investigated in 28-days predictive value of entire score in patients with severe acute dyspnea and suspecting to acute HF or ADHF. Although three biomarkers - Mid Pro-ANP, MR-proADM and pro-endothelin - have been independently associated with prognosis of acute and chronic HF regardless LFEF, MR-proADM had improved discriminative value of NPs in combination with copeptin and troponin T. Overall, there is no clarity and consistent evidence for multiple biomarker strategy in improvement in $\mathrm{CV}$ mortality and $\mathrm{CV}$ outcomes. It has been suggesting that sST2, MR-proADM and galectin-3 could improve prognostication of HF-related hospitalization and death, when they are added to NPs.

\section{Conclusions}

There are several controversies regarding importance of predictive value for survival and incremental prognostication in diagnosis of HFrEF and HFpEF. Probably, biomarkers of inflammation and vascular remodeling are predominantly observed in HFpEF, while biomarkers of biomechanical stress and collagen metabolism much more accurately predicted clinical outcome in HFrEF. All these require improving clinical guideline recommendations for optimizing HF therapy in routine clinical practice under biomarkers' control. There is need in larger clinical trials to head-to-head compare different biomarkers and clear their role in diagnosis and guided therapy of HF.

\section{References}

1. Ponikowski P, Voors AA, Anker SD, Bueno H, Cleland JG, et al. (2016) 2016 ESC guidelines for the diagnosis and treatment of acute and chronic heart failure: the Task Force for the diagnosis and treatment of acute and chronic heart failure of the European Society of Cardiology (ESC) developed with the special contribution of the Heart Failure Association (HFA) of the ESC. Eur Heart J 37: 2129-2200.

2. Wettersten N, Maisel AS (2016) Biomarkers for Heart Failure: An Update for Practitioners of Internal Medicine. Am J Med 129: 560-567.

3. Ledwidge M, Gallagher J, Conlon C, Tallon E, O'Connell E, et al. (2013) Natriuretic peptide-based screening and collaborative care for heart failure: the STOP-HF randomized trial. JAMA 310: 66-74.

4. Chow SL, Maisel AS, Anand I, Bozkurt B, de Boer RA et al (2017) Role of Biomarkers for the Prevention, Assessment, and Management of Heart Failure: A Scientific Statement From the American Heart Association. Circulation 135: e1054-e1091.

5. Yancy CW, Jessup M, Bozkurt B, Butler J, Casey DE Jr, et al. (2017) 2017 ACC/AHA/HFSA Focused Update of the 2013 ACCF/AHA Guideline for the Management of Heart Failure: A Report of the American College of Cardiology/ American Heart Association Task Force on Clinical Practice Guidelines and the Heart Failure Society of America. Circulation 136.

6. Boulogne M, Sadoune M, Launay JM, Baudet M, Cohen-Solal A, et al. (2017) Inflammation versus mechanical stretch biomarkers over time in acutely decompensated heart failure with reduced ejection fraction. Int J Cardiol 226 53-59.

7. Souza BSF, Silva DN, Carvalho RH, Sampaio GLA, Paredes BD, et al. (2017) Association of Cardiac Galectin-3 Expression, Myocarditis, and Fibrosis in Chronic Chagas Disease Cardiomyopathy. Am J Pathol 187: 1134-1146.

8. Lala RI, Lungeanu D, Darabantiu D, Pilat L, Puschita M (2017) Galectin-3 as a marker for clinical prognosis and cardiac remodeling in acute heart failure. Herz, pp: 1-10

9. Imran TF, Shin HJ, Mathenge N, Wang F, Kim B, et al. (2017) Meta-Analysis of the Usefulness of Plasma Galectin-3 to Predict the Risk of Mortality in Patients With Heart Failure and in the General Population. Am J Cardiol 119: 57-64.

10. Besler C, Lang D, Urban D, Rommel KP, von Roeder M, et al. (2017) Plasma and Cardiac Galectin-3 in Patients With Heart Failure Reflects Both Inflammation and Fibrosis: Implications for Its Use as a Biomarker. Circ Heart Fail 10: e003804.

11. Srivatsan V, George M, Shanmugam E (2015) Utility of galectin-3 as a prognostic biomarker in heart failure: where do we stand? Eur J Prev Cardiol 22: $1096-110$.

12. Boulogne M, Sadoune M, Launay JM, Baudet M, Cohen-Solal A, et al. (2017) Inflammation versus mechanical stretch biomarkers over time in acutely decompensated heart failure with reduced ejection fraction. Int J Cardiol 226: 53-59.

13. Billebeau G, Vodovar N, Sadoune M, Launay JM, Beauvais F, et al. (2017) Effects of a cardiac rehabilitation programme on plasma cardiac biomarkers in patients with chronic heart failure. Eur J Prev Cardiol 24: 1127-1135.

14. Bayes-Genis A, de Antonio M, Vila J, Peñafiel J, Galán A, et al. (2014) Head-tohead comparison of 2 myocardial fibrosis biomarkers for long-term heart failure risk stratification: ST2 versus galectin-3. J Am Coll Cardiol 63: 158-166.

15. Anguita M (2012) High-sensitivity troponins and prognosis of heart failure. Rev Clin Esp 217: 95-96.

16. Nagarajan V, Hernandez AV, Tang WH (2012) Prognostic value of cardiac troponin in chronic stable heart failure: a systematic review. Heart 98: 1778-1786.

17. Masson S, Latini R, Anand IS (2010)An update on cardiac troponins as circulating biomarkers in heart failure. Curr Heart Fail Rep 7: 15-21.

18. Chmurzynska A (2006) The multigene family of fatty acidbinding proteins (FABPs): function, structure, and polymorphism. J Appl Genet 47: 39-48.

19. Qian HY, Huang J, Yang YJ, Yang YM, Li ZZ, et al. (2016) Heart-type Fatty Acid Binding Protein in the Assessment of Acute Pulmonary Embolism. Am J Med Sci 352: 557-562.

20. Kitai T, Kim YH, Kiefer K, Morales R, Borowski AG, et al. (2017) Circulating intestinal fatty acid-binding protein (I-FABP) levels in acute decompensated heart failure. Clin Biochem 50: 491-495.

21. Malek V, Gaikwad AB (2017) Neprilysin inhibitors: A new hope to halt the diabetic cardiovascular and renal complications? Biomed Pharmacother 90: 752-759.

22. Wong PC, Guo J, Zhang A (2017) The renal and cardiovascular effects of natriuretic peptides. Absence of clear clinical recommendations of biomarkerbased HF therapy is the main cause of uncertainty regarding practical use of this approach. Adv Physiol Educ 41: 179-185.

23. Luchner A, von Haehling S, Holubarsch C, Keller T, Knebel F, et al. (2017) Indications and Clinical Implications of the Use of the Cardiac Markers BNP and NT-proBNP. Dtsch Med Wochenschr 142: 346-355.

24. Aspromonte N, Gulizia MM, Clerico A, Di Tano G, Emdin M, et al. (2016) ANMCO/ELAS/SIBioC Consensus document: Recommendations for the use of cardiac biomarkers in heart failure patients]. G Ital Cardiol (Rome) 17: 615-656.

25. Nakanishi M, Nakao K, Kumasaka L, Arakawa T, Fukui S, et al. (2017) Improvement in Exercise Capacity by Exercise Training Associated With Favorable Clinical Outcomes in Advanced Heart Failure With High B-Type Natriuretic Peptide Level. Circ J. 